\title{
Synchronous Behavior for Memristive Synapse-Connected Chay Twin-Neuron Network and Hardware Implementation
}

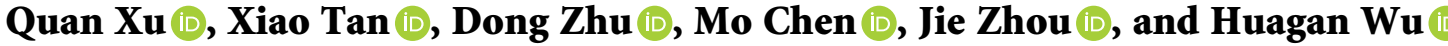 \\ School of Information Science and Engineering, Changzhou University, Changzhou 213164, China \\ Correspondence should be addressed to Huagan Wu; wuhg@cczu.edu.cn
}

Received 11 July 2020; Accepted 17 August 2020; Published 11 September 2020

Guest Editor: Viet-Thanh Pham

Copyright (c) 2020 Quan Xu et al. This is an open access article distributed under the Creative Commons Attribution License, which permits unrestricted use, distribution, and reproduction in any medium, provided the original work is properly cited.

Synchronous behavior can be responsible for the function or dysfunction of a neural network. To employ a memristor with threshold memductance as a bidirectional synapse, a memristive synapse-connected Chay twin-neuron network is constructed. This paper numerically presents the synchronous behavior for four representative firing activities in the memristive twin-neuron network by utilizing time-domain waveforms, synchronized transition states (STSs), and mean synchronization errors (MSEs). Indeed, the synchronous behaviors are truly related to the coupling strength and initial condition of the memristor. Besides, utilizing the powerful XC7Z020 FPGA, a digitally circuit-implemented electro-neuron and the memristive synapse-connected Chay twin-neuron network are made. Thereafter, the four representative firing activities and their STSs are experimentally captured to further confirm the numerical simulations.

\section{Introduction}

Biological system propagates and handles neural signal via abundant collective behaviors [1-5]. As one of the most outstanding collective behaviors in neuronal network, synchronous behavior is important for the realization of signal propagation, information handling, and other activities [6-8]. Synchronous behavior for neuron-coupled models abstracted from the biological system has attracted much interest due to their simplicity and without loss of generality [9-15]. Numerous investigations of synchronous behavior in gap junction-coupled Huber-Braun neurons [9], gap junction-coupled or noise-sustained FitzHugh-Nagumo neurons [10-12], feedback unidirectional-coupled Hindmarsh-Rose neurons [7, 13], and chemical synaptic-coupled Hindmarsh-Rose neurons [14] have been explored in theoretical and numerical levels. Besides, excessive synchronization can be achieved through stimulating neuron by external control signal, which can be employed to cure some disorder diseases of Alzheimer's, epilepsy, Parkinson's, and hand shaking [15-17].

Based on the law of electromagnetic induction theory, Lv et al. have come up with the idea that the memristor can be employed as a connected synapse to describe transmembrane current generated by the membrane potentials' difference between two coupled neurons $[18,19]$. When two coupled neurons own different initial conditions, i.e., different neuron membrane potentials, electromagnetic induction current can be generated to exchange the charged ions across the membrane. In this way, the neuron membrane potentials are altered and synchronization could be achieved [7]. The memristor is a novel circuit device with its memductance really depending on the input and with memory effect [20], which is regarded as a valid candidate for neuronal synapse [21]. Thus, it is interesting to find the emergence of synchronous behavior for memristor synapse-coupled two neurons having different membrane potentials between them [22-25]. The coexisting firing activities and synchronous behaviors associated to initial condition of the memristor in a memristive synapse-connected Morris-Lecar double neurons' network are explored [22]. Besides, the coexisting firing activities and initial-associating bifurcation behaviors, as well as the extreme events are disclosed in two adjacent Hindmarsh-Rose neurons connected by flux-controlled memristor [26]. However, the memristor initial condition-associated synchronous behavior still needs further investigation.

Three-dimensional (3D) Chay neuron model is a more physiologically excitable three-variable neuron model with simple mathematical form and rich essential features of electrical activities [27]. In essence, the 3D Chay neuron 
model has the feature of a fast-slow structure to represent the membrane potential and ionic events in excitable membranes as in [28]. The 3D Chay neuron model can give birth to abundant firing activities of periodic and chaotic bursting/ spiking behaviors [29], as well as the bifurcation scenarios for stochastic resonance are disclosed [30]. Besides, GWN and magnetro-acoustical simulation-induced multiple firing activities are explored by numerical simulations [31-33]. From the viewpoint of ions' effect mechanisms in biological neuron, the outward current carried by $\mathrm{K}^{+}$ions and the inward one by $\mathrm{Na}^{+}$and $\mathrm{Ca}^{2+}$ ions play key role in living biological activities. Thus, beyond all these investigations, by selecting four sets of the maximal conductances for the two ions currents, four representative firing activities are revealed. Besides, the synchronization for these four representative firing activities in coupled Chay neuron models are barely disclosed, which hinders the process of unveiling living biological activities.

The theoretical explorations and numerical simulations are classical methods to investigate neural dynamics, but hardware implementations and experimental measurements have become increasingly promising for diverse neuron-based engineering applications [34-43]. To date, on the benefit of realtime modification, easy software control, and adjustment, field programmable gate array (FPGA) has been employed to verify the dynamical behaviors of Hindmarsh-Rose neuron model [35, 36], Wilson neuron model [37], Morris-Lecar neuron model [38], and FitzHugh-Nagumo neuron model [39] in the hardware level. One of another remarkable benefit is that the FPGA-based experiments can assign the desired initial conditions in software accurately and easily, which is conducive to investigate initial condition-associated electrical activities in the hardware level [40]. To consider the virtue of FPGA-based realization method, there should be important significance to develop various digital electronic neurons for utilizing in hardware level synthesizers for large-scale neuromprphic circuits. Besides, the digitally circuit-implemented neuron network can effectively imitate the synchronous behavior in the network, which can promote the integrated circuit design and diverse neuron-based engineering applications [22]. To our best knowledge, there is no comprehensive exploration about the Chay neuron model in this valuable approach.

The arrangement of this paper is well designed as follows. The representative firing activities in a $3 \mathrm{D}$ Chay neuron model are briefly reviewed by employing numerical plots of phase trajectories and time-domain waveforms. Besides, a memristive synapse-connected Chay twin-neuron network is built in Section 2. Section 3 explores synchronous behavior in the memristive Chay twin-neuron network by utilizing time-domain waveforms, STSs, and MSEs. A FPGA-based electronic neuron and the memristive synapse-connected Chay twin-neuron network are realized and the hardware tests are executed to verify the numerically simulated ones in Section 4. Eventually, the conclusion is drawn.

\section{Memristive Synapse-Connected Chay Twin- Neuron Network}

2.1. Minireview for 3D Chay Neuron Model. The 3D Chay neuron model is an effective candidate in the numerical simulation for electrical activities in single biological neuron [27], which is described as

$$
\begin{aligned}
\frac{\mathrm{d} V}{\mathrm{~d} t} & =g_{1} m_{\infty}^{3} h_{\infty}\left(V_{I}-V\right)+g_{K, V} n^{4}\left(V_{K}-V\right)+g_{K, C} \frac{C}{1+C}\left(V_{K}-V\right)+g_{L}\left(V_{L}-V\right), \\
\frac{\mathrm{d} n}{\mathrm{~d} t} & =\frac{\left(n_{\infty}-n\right)}{\tau_{n}}, \\
\frac{\mathrm{d} C}{\mathrm{~d} t} & =\rho\left[m_{\infty}^{3} h_{\infty}\left(V_{C}-V\right)-k_{C} C\right],
\end{aligned}
$$

where the three dynamic variables $V, n$, and $C$ correspond to the membrane potential, open probability of the voltagesensitive potassium ions channel, and intracellular concentration of calcium ions, respectively. The model parameter in (1) is assigned and shown in Table 1. Besides, $y_{\infty}=\alpha_{y} /\left(\alpha_{y}+\beta_{y}\right)$ is unified to the expressions of $m_{\infty}, h_{\infty}$, and $n_{\infty}$, in which $y$ presents the alphabets $m, h$, and $n$. Herein, the expression formats for $\alpha_{m}, \beta_{m}, \alpha_{h}, \beta_{h}, \alpha_{n}, \beta_{n}$, and $\tau_{n}$ are 


$$
\begin{aligned}
& \alpha_{m}=\frac{0.1(25+V)}{\left(1-e^{-(V+25 / 10)}\right)} \\
& \beta_{m}=4 e^{-(V+50 / 18)}, \\
& \alpha_{h}=0.07 e^{-(V+50 / 20)}, \\
& \beta_{h}=\frac{1}{\left(1+e^{-(V+20 / 10)}\right)} \\
& \alpha_{n}=\frac{0.01(20+V)}{\left(1-e^{-(V+20 / 10)}\right)} \\
& \beta_{n}=0.125 e^{-(V+30 / 80)}, \\
& \tau_{n}=\frac{1}{\left[r_{n}\left(\alpha_{n}+\beta_{n}\right)\right]} .
\end{aligned}
$$

On account of the fast-slow effect, the bursting and spiking behaviors are two representative firing activities generated in this neuron model [27]. By using MATLAB ODE45-based method with time step $10^{-3} \mathrm{~s}$, fixing the initial conditions $(0.1 \mathrm{mV}, 0.1,0.1 \mathrm{nmol} / \mathrm{L})$, and employing the model parameters in Table 1, note that "MaxStep" and "RelTol" in MATLAB-based ODE45 algorithm should be assigned as $10^{-3} \mathrm{~s}$ and $10^{-4} \mathrm{~s}$, respectively.

The firing activities related to the reversal potentials $[30,32]$, external stimulus $[31,33]$, and time kinetic constant [41] have been investigated in numerical survey. Differently, by selecting four sets of model parameters for the maximal conductance $g_{\mathrm{I}}$ of mixed $\mathrm{Na}^{+}-\mathrm{Ca}^{2+}$ channel and maximal conductance $g_{\mathrm{K}, \mathrm{V}}$ of potassium ions channel, four representative types of firing activities including periodic and chaotic bursting and chaotic spiking behaviors are revealed by utilizing the trajectories in the $C-V$ plane and the timedomain waveforms of the membrane potential $V$ and intracellular concentration $C$ of calcium ions, as shown in Figure 1. Figures 1(a) and 1(d) exhibit two kinds of periodic bursting behaviors, Figure 1(b) demonstrates chaotic bursting behavior with disordered spikes, and Figure 1(c) illustrates conventional chaotic spiking behavior. The bifurcation mechanisms for the two kinds of periodic bursting behaviors are different. Those are periodic fold/fold bursting in Figure 1(a) and periodic fold/homoclinic bursting in Figure 1(d) [42, 43]. Figure 2(b) shows the chaotic fold/ homoclinic bursting behaviors, and Figure 1(c) denotes the chaotic spiking activity. The synchronous behaviors in a memristive synapse-connected Chay twin-neuron network under the four sets of model parameters will be disclosed in the next sections, respectively.

2.2. Modeling for Memristive Synapse-Connected Twin-Neuron Network. Support two identical neurons are bidirectionally coupled by memristor synapse to represent the electromagnetic induction effect induced with the membrane potential differences between them. Herein, the memristor with threshold memductance $W(\varphi)=\tan \mathrm{h}(\varphi)$ and the membrane potential difference $V_{M}=V_{1}-V_{2}$ are employed [26], so the inducted current $I_{M}$ and memristor synapse characteristic model can be described as

$$
\begin{aligned}
I_{M} & =W(\varphi) V_{M}=\tanh (\varphi)\left(V_{1}-V_{2}\right), \\
\dot{\varphi} & =V_{M}=V_{1}-V_{2},
\end{aligned}
$$

with two identical Chay neurons coupled by the threshold memristor, and a memristive synapse-connected Chay twinneuron network can be described as

$$
\begin{aligned}
\frac{\mathrm{d} V_{1}}{\mathrm{~d} t} & =g_{\mathrm{I}} m_{1 \infty}^{3} h_{1 \infty}\left(V_{\mathrm{I}}-V_{1}\right)+g_{\mathrm{K}, \mathrm{V}} n_{1}^{4}\left(V_{\mathrm{K}}-V_{1}\right)+g_{\mathrm{K}, \mathrm{C}} \frac{C_{1}}{1+C_{1}}\left(V_{\mathrm{K}}-V_{1}\right)+g_{\mathrm{L}}\left(V_{\mathrm{L}}-V_{1}\right)+k \tanh \varphi\left(V_{1}-V_{2}\right), \\
\frac{\mathrm{d} n_{1}}{\mathrm{~d} t} & =\frac{\left(n_{1 \infty}-n_{1}\right)}{\tau_{1 n}}, \\
\frac{\mathrm{d} C_{1}}{\mathrm{~d} t} & =\rho\left[m_{1 \infty}^{3} h_{1 \infty}\left(V_{\mathrm{C}}-V_{1}\right)-k_{\mathrm{C}} C_{1}\right], \\
\frac{\mathrm{d} V_{2}}{\mathrm{~d} t} & =g_{\mathrm{I}} m_{2 \infty}^{3} h_{2 \infty}\left(V_{\mathrm{I}}-V_{2}\right)+g_{\mathrm{K}, \mathrm{V}} n_{2}^{4}\left(V_{\mathrm{K}}-V_{2}\right)+g_{\mathrm{K}, \mathrm{C}} \frac{C_{2}}{1+C_{2}}\left(V_{\mathrm{K}}-V_{2}\right)+g_{\mathrm{L}}\left(V_{\mathrm{L}}-V_{2}\right)-k \tanh \varphi\left(V_{1}-V_{2}\right), \\
\frac{\mathrm{d} n_{2}}{\mathrm{~d} t} & =\frac{\left(n_{2 \infty}-n_{2}\right)}{\tau_{2 n}}, \\
\frac{\mathrm{d} C_{2}}{\mathrm{~d} t} & =\rho\left[m_{2 \infty}^{3} h_{2 \infty}\left(V_{\mathrm{C}}-V_{2}\right)-k_{\mathrm{C}} C_{2}\right], \\
\frac{\mathrm{d} \varphi}{\mathrm{d} t} & =V_{1}-V_{2},
\end{aligned}
$$


TABLE 1: Parameters' values and significations of the 3D Chay neuron model.

\begin{tabular}{lcc}
\hline Parameters & Significations & Values \\
\hline$V_{\mathrm{I}}$ & Reversal potentials for mixed $\mathrm{Na}^{+}-\mathrm{Ca}^{2+}$ ions & $100 \mathrm{mV}$ \\
$V_{\mathrm{K}}$ & Reversal potentials for $\mathrm{K}^{+}$ions & $-75 \mathrm{mV}$ \\
$V_{\mathrm{L}}$ & Reversal potentials for leakage ions & $-40 \mathrm{mV}$ \\
$V_{\mathrm{C}}$ & Reversal potentials for Ca $\mathrm{Ca}^{2+}$ ions & $100 \mathrm{mV}$ \\
$g_{\mathrm{I}}$ & Maximal conductance of mixed $\mathrm{Na}^{+}-\mathrm{Ca}^{2+}$ channel & $1925 \mathrm{mS} / \mathrm{cm}^{2}$ \\
$g_{\mathrm{K}, \mathrm{V}}$ & Maximal conductance of $\mathrm{K}^{+}$channel & $1700 \mathrm{mS} / \mathrm{cm}^{2}$ \\
$g_{\mathrm{K}, \mathrm{C}}$ & Maximal conductance of Ca ${ }^{2+}$-sensitive $\mathrm{K}^{+}$channel & $12 \mathrm{mS}_{\mathrm{cm}^{2}}$ \\
$g_{\mathrm{L}}$ & Maximal conductance of leakage channel & $7 \mathrm{mS} / \mathrm{cm}^{2}$ \\
$r_{n}$ & Time kinetic constant of the fast variable & $230 \mathrm{~ms}$ \\
$k_{\mathrm{C}}$ & Rate constant for the efflux of intracellular Ca ${ }^{2+}$ ions & $3.3 / 18 \mathrm{~ms}{ }^{-1}$ \\
$\rho$ & Time constant determines the changing rate C & $0.27 \mathrm{~ms}$ \\
\hline
\end{tabular}
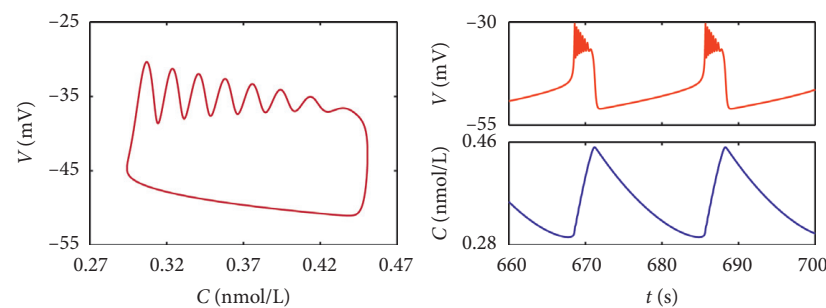

(a)
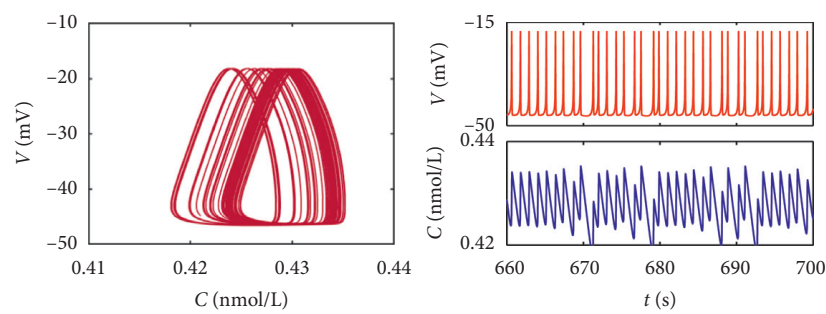

(c)
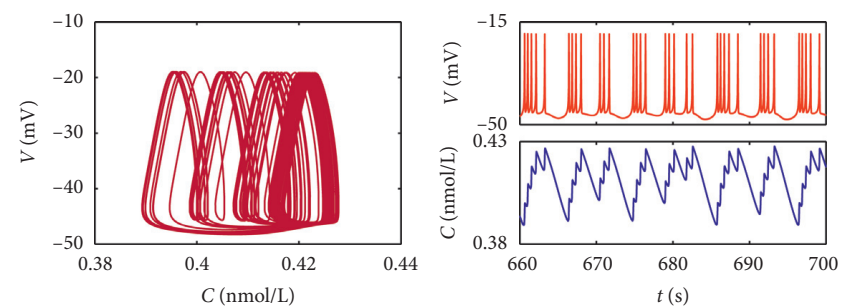

(b)
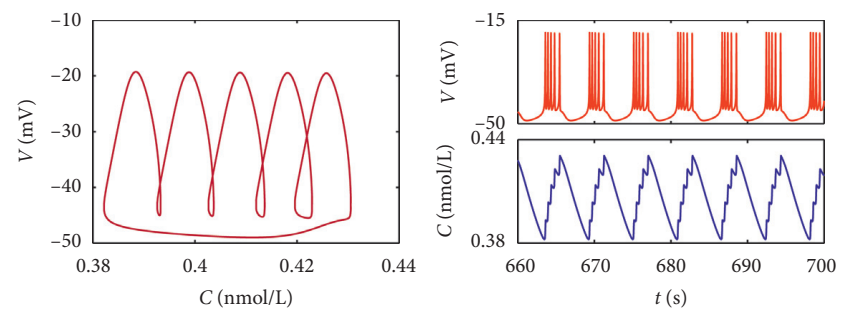

(d)

Figure 1: Trajectories in the $C-V$ plane and time-domain waveforms of $V$ and $C$ for different $g_{\mathrm{I}}$ and $g_{\mathrm{K}, \mathrm{V}}$. (a) Periodic bursting behavior for $g_{\mathrm{I}}=1250 \mathrm{mS} / \mathrm{cm}^{2}$ and $g_{\mathrm{K}, \mathrm{V}}=1700 \mathrm{mS} / \mathrm{cm}^{2}$; (b) chaotic bursting behavior for $g_{\mathrm{I}}=1850 \mathrm{mS} / \mathrm{cm}^{2}$ and $g_{\mathrm{K}, \mathrm{V}}=1700 \mathrm{mS} / \mathrm{cm}^{2}$; (c) chaotic spiking behavior for $g_{\mathrm{I}}=1925 \mathrm{mS} / \mathrm{cm}^{2}$ and $g_{\mathrm{K}, \mathrm{V}}=1700 \mathrm{mS} / \mathrm{cm}^{2}$; (d) periodic bursting behavior with 5 spikes per burst for $g_{\mathrm{I}}=1800 \mathrm{mS} / \mathrm{cm}^{2}$ and $g_{\mathrm{K}, \mathrm{V}}=1650 \mathrm{mS} / \mathrm{cm}^{2}$.

where $V_{1}$ and $V_{2}$ are the membrane potential, $n_{1}$ and $n_{2}$ are probability of the voltage-sensitive $\mathrm{K}^{+}$channel for the two identical Chay neuron models, $C_{1}$ and $C_{2}$ are the intracellular concentration of calcium ions for them, respectively, and $k \tanh \varphi\left(V_{1}-V_{2}\right)$ stands for the memristor synapsecoupled item with $k$ as positive to feature the coupling strength. In (4), the coupling strength $k$ can control the effect of memristor synapse. Actually, the memristor synapseconnected item just inputs additive current leading to that the membrane potentials which can be regulated. Also, (4) explained the mechanism for memristive feedback control. Besides, $y_{i \infty 0}=\alpha_{i y} /\left(\alpha_{i y}+\beta_{i y}\right)$ can be unified to the explications of $m_{i \infty}, h_{i \infty}$, and $n_{i \infty}$, in which $y$ stands for $m, h$, and $n$ and $i=1,2$. The explicit expressions for $\alpha_{i m}, \beta_{i m}, \alpha_{i h}, \beta_{i h}, \alpha_{i n}, \beta_{i n}$, and $\tau_{\text {in }}$ are

$$
\begin{aligned}
& \alpha_{i m}=\frac{0.1\left(25+V_{i}\right)}{\left(1-e^{-\left(V_{i}+25 / 10\right)}\right)}, \beta_{i m}=4 e^{-\left(V_{i}+50 / 18\right)}, \\
& \alpha_{i h}=0.07 e^{-\left(V_{i}+50 / 20\right)}, \beta_{i h}=\frac{1}{\left(1+e^{-\left(V_{i}+20 / 10\right)}\right)}, \\
& \alpha_{i n}=\frac{0.01\left(20+V_{i}\right)}{\left(1-e^{-\left(V_{i}+20 / 10\right)}\right)}, \beta_{i n}=0.125 e^{-\left(V_{i}+30 / 80\right)}, \\
& \tau_{\text {in }}=\frac{1}{\left[r_{n}\left(\alpha_{i n}+\beta_{i n}\right)\right]} .
\end{aligned}
$$



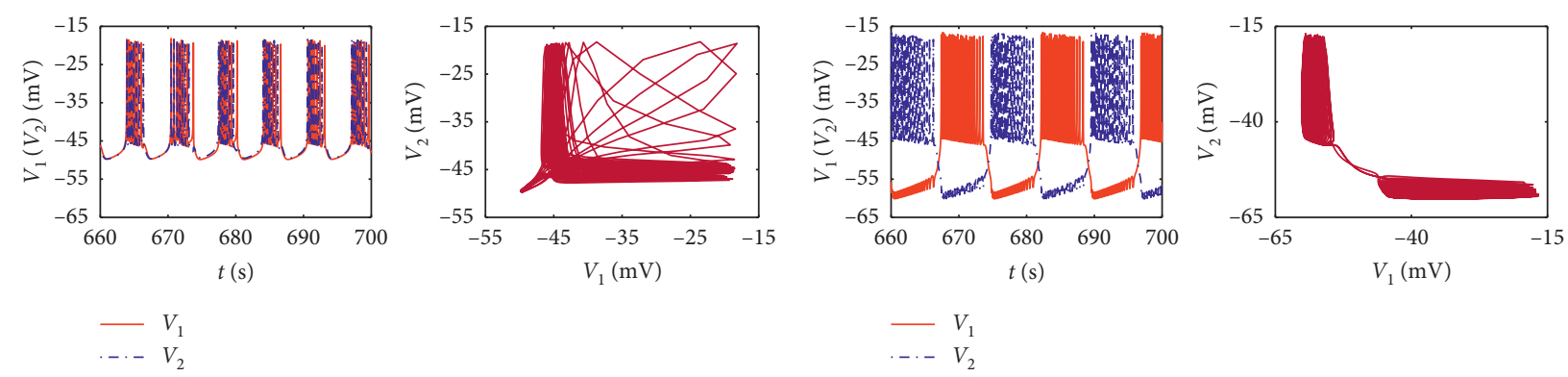

(a)
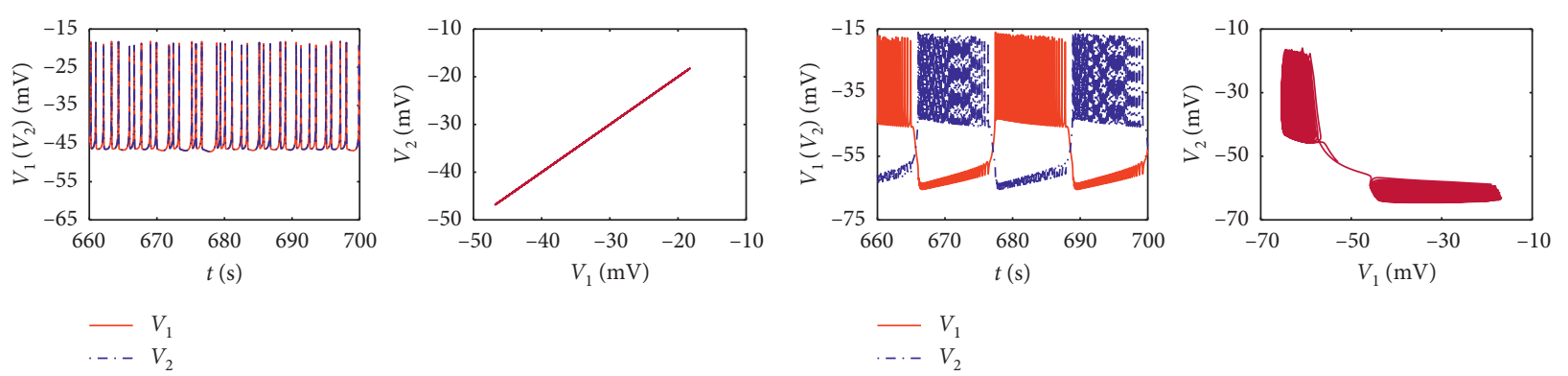

(c)

(d)

FIGURE 2: Synchronous behavior for the memristive twin-neuron network with various $k$ and $\varphi_{0}$, where the lefts are time-domain waveforms and the rights are STSs. (a) $k=1$ and $\varphi_{0}=-2 \mathrm{mWb}$; (b) $k=3$ and $\varphi_{0}=-2 \mathrm{mWb}$; (c) $k=4$ and $\varphi_{0}=-2 \mathrm{mWb}$; (d) $k=4$ and $\varphi_{0}=1 \mathrm{mWb}$.

Thus, the synchronous behaviors for the memristive synapse-connected Chay twin-neuron network can be disclosed by system (4). Following on this, we mainly focus on exploring the memristor initial condition and coupling strength associated synchronous behaviors in the proposed memristive network.

\section{Synchronous Behaviors for the Chay Twin- Neuron Network}

The exploration of synchronous behaviors for the Chay twin-neuron network is performed by MATLAB numerical simulations. Herein, we mainly focus on the two coupled neurons with only difference initial membrane potentials between them. Thus, the Chay twin-neuron network is triggered by initial conditions $(0.1 \mathrm{mV}, 0,0.1 \mathrm{nmol} / \mathrm{L}, 1 \mathrm{mV}$, $\left.0,0.1 \mathrm{nmol} / \mathrm{L}, \varphi_{0}\right)$ without loss of the generality, within which the memristor initial condition $\varphi_{0}$ is tunable.

The electrical activity is chaotic spiking under the model parameters in Table 1, for each neuron in the memristive synapse-connected Chay twin-neuron network. The synchronized transition states (STSs) are plotted at the right in Figures 2(a)-2(d). There exist no errors between the two membrane potentials with a line in the $V_{1}-V_{2}$ plane, which means that the two neurons are in sync. Otherwise, the relatively large errors between the two membrane potentials indicate the two neurons out of synchronization. When $\varphi_{0}=-2 \mathrm{mWb}$, the difference between the two membrane potentials become smaller with $k=1$ and 3 increasingly and disappear with $k=4$, as shown in Figures 2(a)-2(c). This manifests that the two coupled neurons are asymptotically synchronized with increasing $k$. However, when $k=4$ with increasing $\varphi_{0}$ as $-2 \mathrm{mWb}$ and $1 \mathrm{mWb}$, the difference between the two membrane potentials become larger, as shown in Figures 2(c) and 2(d). It is indicated that the two coupled neurons are loose their synchronization. Summarily, by increasing the coupling strength $k$ or decreasing memristor initial condition $\varphi_{0}$, the synchronization can be realized and the difference between the two membrane potentials is becoming smaller in the memristive synapse-connected Chay twin-neuron network. The mechanism for this process is that the coupling memristor exchanges the magnetic flux. Thus, the induced current is the carrier to drive the two Chay neurons in sync. Otherwise, the two connected Chay neurons are loss synchronization under tiny electromagnetic induction outputs.

To fully explore the synchronous behavior associated to the coupling strength $k$ and initial condition $\varphi_{0}$ of the memristor synapse simultaneously, mean synchronization error (MSE) $E$ is employed and defined as

$$
E=\frac{1}{N} \sum_{j=1}^{N} \frac{\sqrt{\left[V_{1}(j)-V_{2}(j)\right]^{2}+\left[n_{1}(j)-n_{2}(j)\right]^{2}+\left[C_{1}(j)-C_{2}(j)\right]^{2}}}{\sqrt{V_{1}(j)^{2}+V_{2}(j)^{2}+n_{1}(j)^{2}+n_{2}(j)^{2}+C_{1}(j)^{2}+C_{2}(j)^{2}}},
$$

where $V_{i}(j), n_{i}(j)$, and $C_{i}(j)(i=1,2)$ are the $j$ th sampling values and $N$ is the number of total samples. The normalized MSE of the Chay twin-neuron network is becoming zero for synchronous state and nonzero for out of synchronization.

Herein, the time sequence interval (600 s and $700 \mathrm{~s}$ ) and the time step $0.01 \mathrm{~s}$ are selected. Thus, the number of total samples is $N=10000$. The normalized MSEs for different coupling strength $k$ and initial condition $\varphi_{0}$, as well as different $g_{\mathrm{I}}$ and $g_{\mathrm{K}, \mathrm{V}}$ are given in the $\varphi_{0}-k$ plane, as plotted 
in Figure 3. The regions padded by red indicate the two connected Chay neurons in sync with $E=0$, whereas the regions padded by other colors denote the two connected Chay neurons loss synchronization with $E>0$. In general, as coupling strength $k$ becoming larger and more negative initial condition $\varphi_{0}$, the normalized MSEs $E$ drops near zero. Thus, the two connected Chay neurons become sync. Contrarily, the two connected Chay neurons are in asynchronous state with $k$ becoming smaller and small negative or positive $\varphi_{0}$.

The normalized MSEs is given in Figure 3. The results show that the synchronous behaviors for the memristive synapse-connected Chay twin-neuron network are really associated with the memristor synapse coupling strength and the initial condition. Such an initial condition related synchronous behavior has been rarely reported in previous literature [22].

\section{FPGA-Based Hardware Implementation}

It is more complex to physically realize the memristive synapse-connected Chay twin-neuron network than a single 3D Chay neuron model by FPGA. Thus, only the memristive synapse-connected Chay twin-neuron model by the digital electronic platform is demonstrated with representativeness. For this aim, fourth-order Runge-Kutta algorithm is utilized to obtain the discrete-time form for model (4) and given as

$$
\begin{aligned}
& \left(V_{1}\right)_{N}=\left(V_{1}\right)_{N-1}+\frac{l\left[D_{1} k_{\left(V_{1}\right) 1}+D_{2} k_{\left(V_{1}\right) 2}+D_{3} k_{\left(V_{3}\right)_{3}}+D_{4} k_{\left(V_{4}\right) 4}\right]}{D_{1}+D_{2}+D_{3}+D_{4}}, \\
& \left(n_{1}\right)_{N}=\left(n_{1}\right)_{N-1}+\frac{l\left[D_{1} k_{\left(n_{1}\right) 1}+D_{2} k_{\left(n_{1}\right) 2}+D_{3} k_{\left(n_{3}\right) 3}+D_{4} k_{\left(n_{1}\right) 4}\right]}{D_{1}+D_{2}+D_{3}+D_{4}}, \\
& \left(C_{1}\right)_{N}=\left(C_{1}\right)_{N-1}+\frac{l\left[D_{1} k_{\left(C_{1}\right) 1}+D_{2} k_{\left(C_{1}\right) 2}+D_{3} k_{\left(C_{1}\right) 3}+D_{4} k_{\left(C_{1}\right) 4}\right]}{D_{1}+D_{2}+D_{3}+D_{4}}, \\
& \left(V_{2}\right)_{N}=\left(V_{2}\right)_{N-1}+\frac{l\left[D_{1} k_{\left(V_{2}\right) 1}+D_{2} k_{\left(V_{2}\right) 2}+D_{3} k_{\left(V_{2}\right) 3}+D_{4} k_{\left(V_{2}\right) 4}\right]}{D_{1}+D_{2}+D_{3}+D_{4}}, \\
& \left(n_{2}\right)_{N}=\left(n_{2}\right)_{N-1}+\frac{l\left[D_{1} k_{\left(n_{2}\right) 1}+D_{2} k_{\left(n_{2}\right) 2}+D_{3} k_{\left(n_{2}\right) 3}+D_{4} k_{\left(n_{2}\right) 4}\right]}{D_{1}+D_{2}+D_{3}+D_{4}}, \\
& \left(C_{2}\right)_{N}=\left(C_{2}\right)_{N-1}+\frac{l\left[D_{1} k_{\left(C_{2}\right) 1}+D_{2} k_{\left(C_{2}\right) 2}+D_{3} k_{\left(C_{2}\right) 3}+D_{4} k_{\left(C_{2}\right) 4}\right]}{D_{1}+D_{2}+D_{3}+D_{4}}, \\
& \varphi_{N}=\varphi_{N-1}+\frac{l\left[D_{1} k_{\varphi 1}+D_{2} k_{\varphi 2}+D_{3} k_{\varphi 3}+D_{4} k_{\varphi 4}\right]}{D_{1}+D_{2}+D_{3}+D_{4}},
\end{aligned}
$$

where $i$ is the sampling interval, $N$ is the total number of iterations, and $D_{q}(q=1,2,3,4)$ denotes the coefficient of variation. To achieve the unique expression of intermediate vector $k_{\left(V_{i}\right)}, k_{\left(n_{i}\right)}, k_{\left(C_{i}\right) q}$, and $k_{\varphi q}(i=1,2$ and $q=1,2,3,4)$, the assumption of $k_{\left(V_{i}\right) 0}=k_{\left(n_{i}\right) 0}=k_{\left(C_{i}\right) q}=k_{\varphi 0}=0$ is applied. Then, one yields

$$
\begin{aligned}
K_{\left(V_{1}\right) q}= & g_{1} m_{N}^{3} h_{N}\left[V_{I}-\left(\left(V_{1}\right)_{N-1}+\frac{l k_{\left(V_{1}\right) q-1}}{D_{q}}\right)\right] \\
& +g_{K, V} n_{1}^{4}\left[V_{K-1}-\left(\left(V_{1}\right)_{N-1}+\frac{l k_{\left(V_{1}\right) q-1}}{D_{q}}\right)\right] \\
& +g_{K, C} \frac{\left(C_{1}\right)_{N-1}}{1+\left(C_{1}\right)_{N-1}} \\
& \cdot\left[V_{K}-\left(\left(V_{1}\right)_{N-1}+\frac{l k_{\left(V_{1}\right) q-1}}{D_{q}}\right)\right] \\
& +g_{L}\left[V_{L}-\left(\left(V_{1}\right)_{N-1}+\frac{l k_{\left(V_{1}\right) q-1}}{D_{q}}\right)\right] \\
& +k \tan \varphi_{N-1}\left(\left(V_{1}\right)_{N-1}+\frac{l k_{\left(V_{1}\right) q-1}}{D_{q}}-\left(V_{2}\right)_{N-1}\right), \\
K_{\left(n_{1}\right) q}= & \frac{d_{N}-\left(\left(n_{1}\right)_{N-1}+\left(l k_{\left(n_{1}\right) q-1} / D_{q}\right)\right)}{\tau_{N}}, \\
K_{\left(C_{1}\right) q}= & \rho\left[m_{N}^{3} h_{N}\left(V_{C}-\left(V_{1}\right)_{N-1}\right)-K_{C}\left(\left(C_{1}\right)_{N-1}+\frac{l k_{\left(C_{1}\right) q-1}}{D_{q}}\right)\right],
\end{aligned}
$$$$
K_{\left(V_{2}\right) q}=g_{I} m_{N}^{3} h_{N}\left[V_{I}-\left(\left(V_{2}\right)_{N-1}+\frac{l k_{\left(V_{2}\right) q-1}}{D_{q}}\right)\right]
$$$$
+g_{K, V} n_{2-1}^{4}\left[V_{K}-\left(\left(V_{2}\right)_{N-1}+\frac{l k_{\left(V_{2}\right) q-1}}{D_{q}}\right)\right]
$$$$
+g_{K, C} \frac{\left(C_{2}\right)_{N-1}}{1+\left(C_{2}\right)_{N-1}}
$$$$
\cdot\left[V_{K}-\left(\left(V_{2}\right)_{N-1}+\frac{l k_{\left(V_{2}\right) q-1}}{D_{q}}\right)\right]
$$$$
+g_{L}\left[V_{L}-\left(\left(V_{2}\right)_{N-1}+\frac{l k_{\left(V_{2}\right) q-1}}{D_{q}}\right)\right]
$$$$
-k \tan \varphi_{N-1}\left(\left(V_{1}\right)_{N-1}-\left(V_{2}\right)_{N-1}-\frac{l k_{\left(V_{2}\right) q-1}}{D_{q}}\right),
$$$$
K_{\left(n_{2}\right) q}=\frac{d_{N}-\left(\left(n_{2}\right)_{N-1}+\left(l k_{\left(n_{2}\right) q-1} / D_{q}\right)\right)}{\tau_{N}},
$$

$$
\begin{aligned}
K_{\left(C_{2}\right) q} & =\rho\left[m_{N}^{3} h_{N}\left(V_{C}-\left(V_{2}\right)_{N-1}\right)-K_{C}\left(\left(C_{2}\right)_{N-1}\right)+\frac{l k_{\left(C_{2}\right) q-1}}{D_{q}}\right], \\
K_{\varphi q} & =\left(V_{1}\right)_{N-1}-\left(V_{2}\right)_{N-1},
\end{aligned}
$$

in which $q=1,2,3,4$, and 


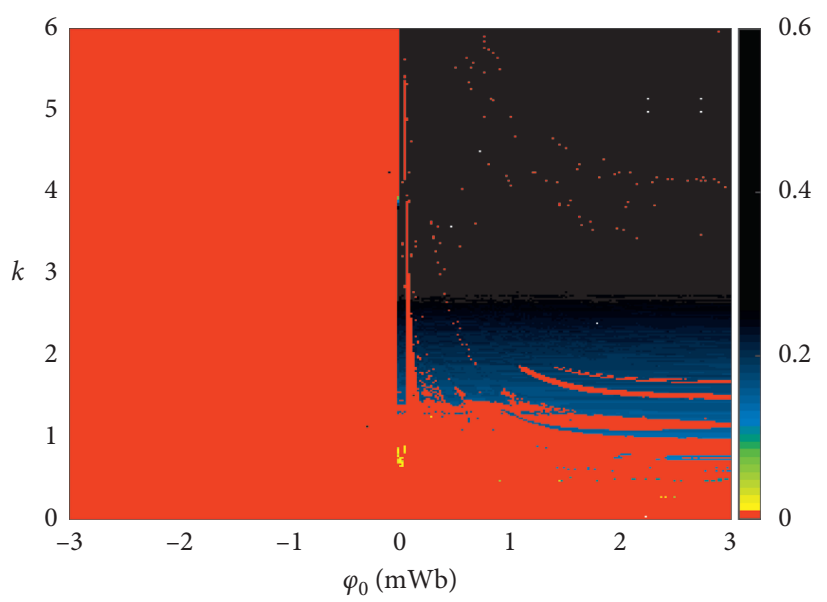

(a)

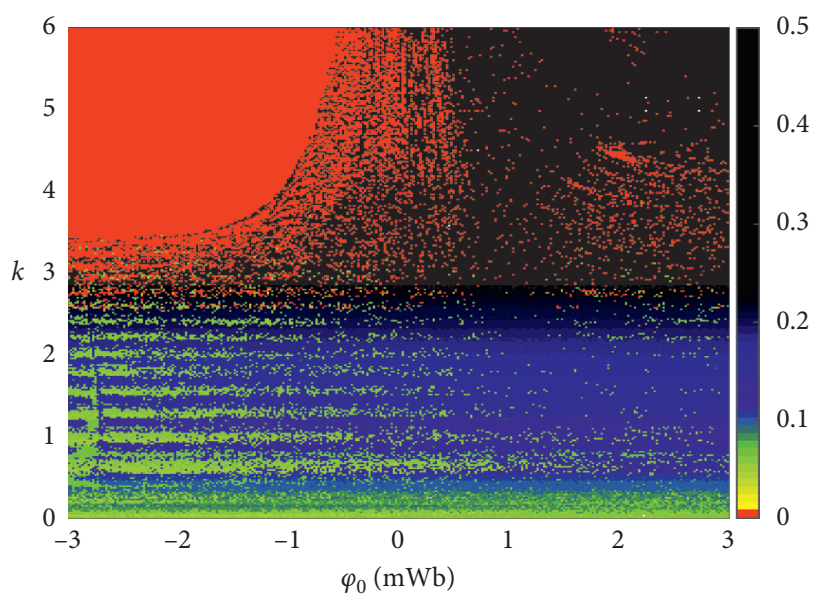

(c)

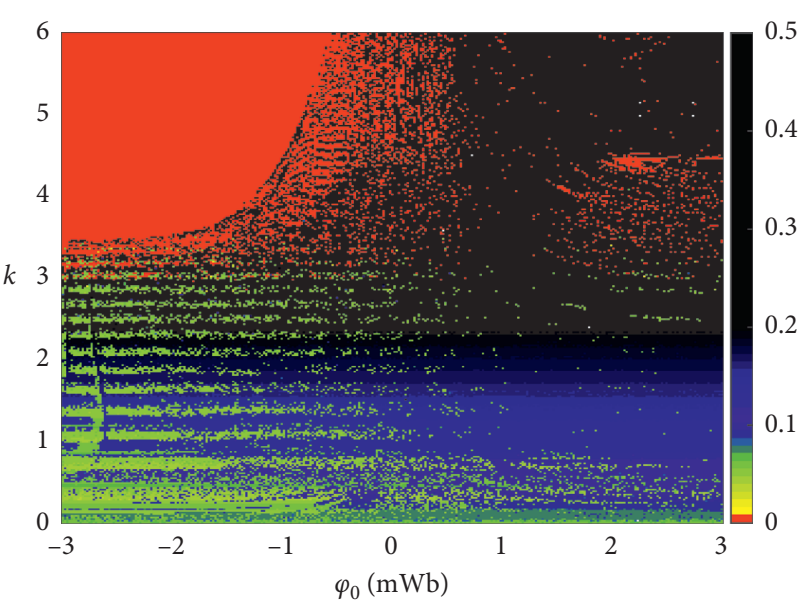

(b)

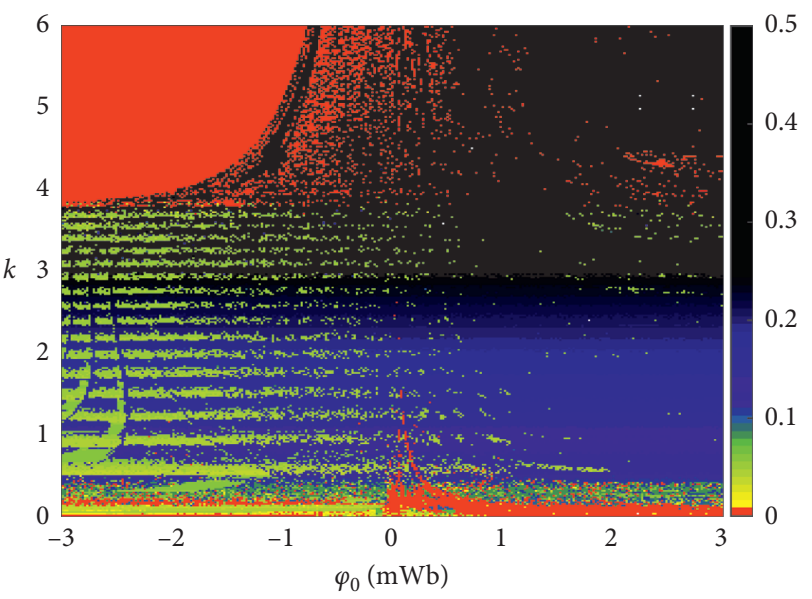

(d)

FIGURE 3: Normalized MSEs of the memristive synapse-connected Chay twin-neuron network in the $\varphi_{0}-k$ plane. (a) $g_{\mathrm{I}}=1250 \mathrm{mS} / \mathrm{cm}^{2}$ and $g_{\mathrm{K}, \mathrm{V}}=1700 \mathrm{mS} / \mathrm{cm}^{2} ;$ (b) $g_{\mathrm{I}}=1850 \mathrm{mS} / \mathrm{cm}^{2}$ and $g_{\mathrm{K}, \mathrm{V}}=1700 \mathrm{mS} / \mathrm{cm}^{2} ;$ (c) $g_{\mathrm{I}}=1925 \mathrm{mS} / \mathrm{cm}^{2}$ and $g_{\mathrm{K}, \mathrm{V}}=1700 \mathrm{mS} / \mathrm{cm}^{2} ;(\mathrm{d}) g_{\mathrm{I}}=1800 \mathrm{mS} / \mathrm{cm}^{2}$ and $g_{\mathrm{K}, \mathrm{V}}=1650 \mathrm{mS} / \mathrm{cm}^{2}$.

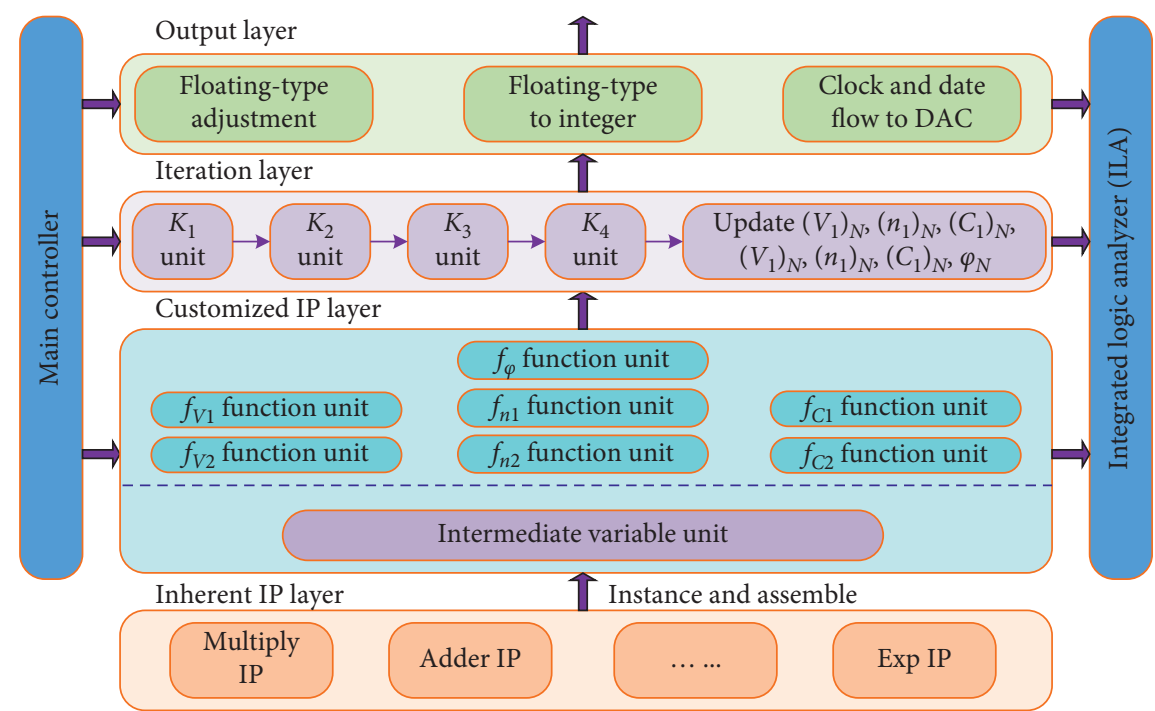

FIgURE 4: Hierarchical structure of the Verilog HDL program for the memristive synapse-connected Chay twin-neuron model. 


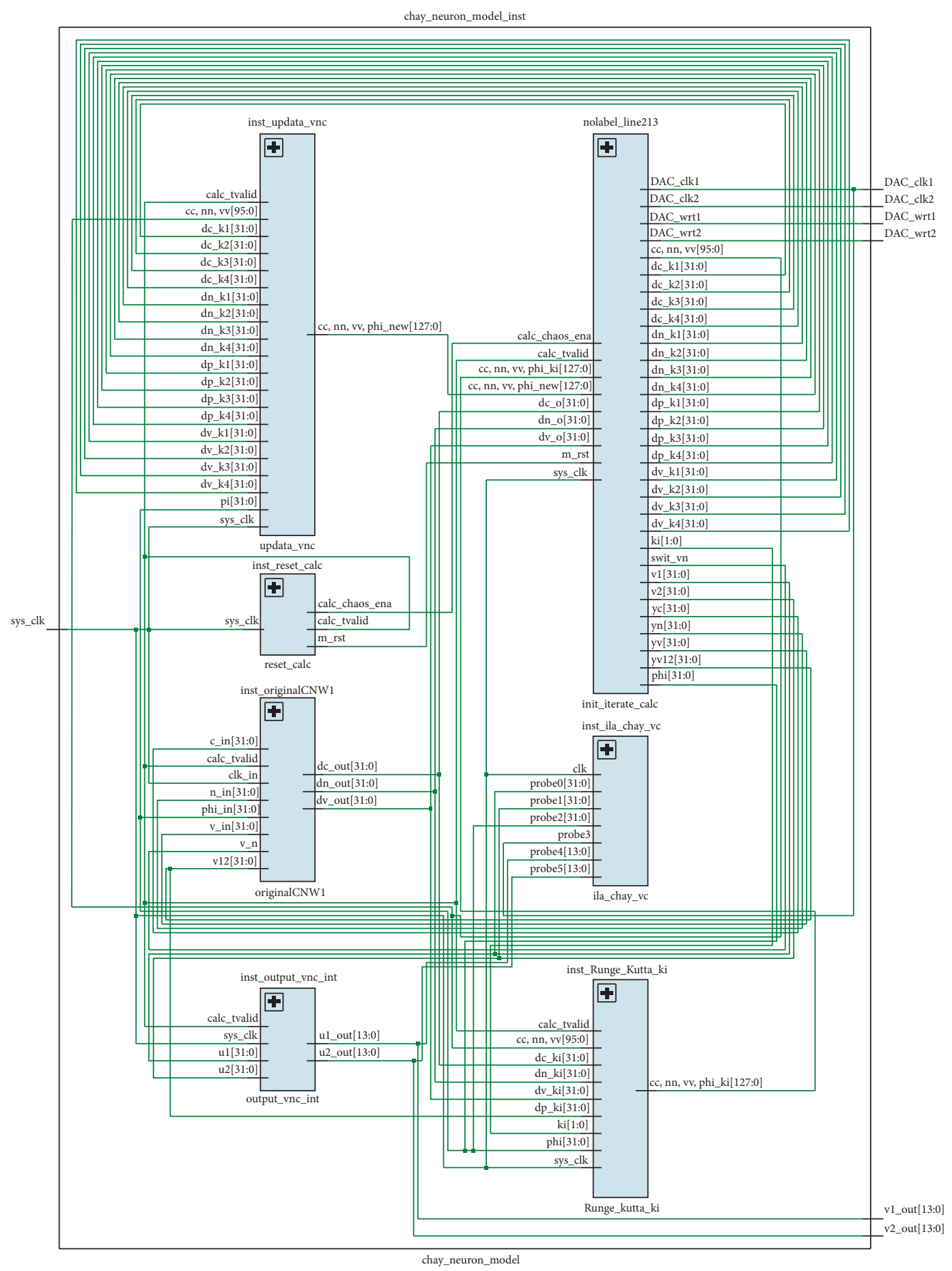

FIGURE 5: RTL schematics of the discrete-time memristive synapse-connected Chay twin-neuron model executed on XC7Z020 FPGA. 

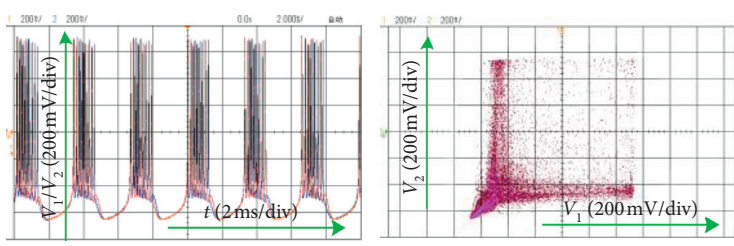

(a)
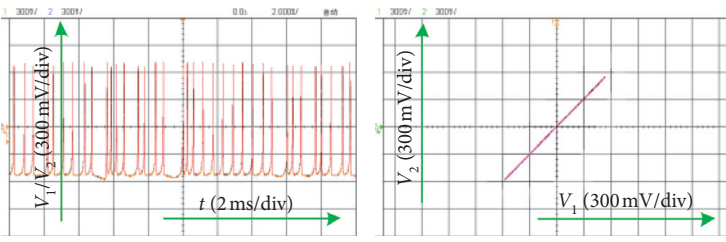

(c)
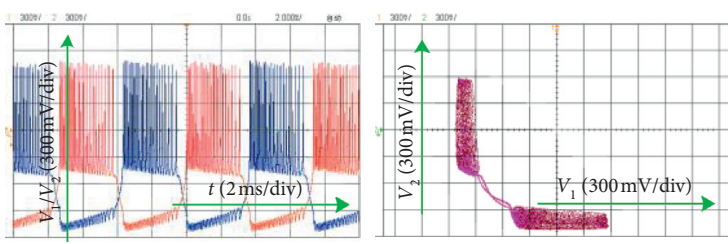

(b)
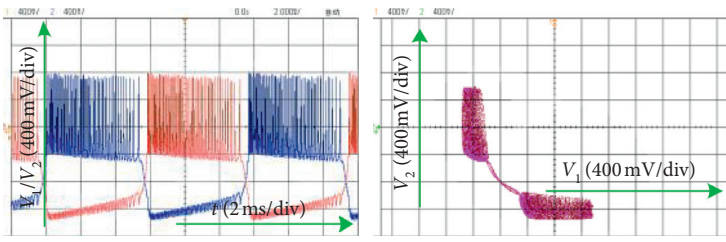

(d)

FIGURE 6: Hardware measured time-domain waveforms and synchronous transition states with different coupling strength $k$ and initial condition $\varphi 0$. (a) $k=1$ and $\varphi 0=-2 \mathrm{mWb}$; (b) $k=3$ and $\varphi 0=-2 \mathrm{mWb}$; (c) $k=4$ and $\varphi 0=-2 \mathrm{mWb}$; (d) $k=4$ and $\varphi 0=1 \mathrm{mWb}$.

$$
\begin{aligned}
& \left(m_{i}\right)_{N}=\frac{25+\left(V_{1}\right)_{N-1}}{25+\left(V_{1}\right)_{N-1}+40\left(1-e^{-0.1\left(V_{i}\right)_{N-1}-2.5}\right) e^{-\left(\left(V_{i}\right)_{N-1}+50\right) / 80}}, \\
& \left(h_{i}\right)_{N}=\frac{7\left(1+{ }^{e-0.1\left(V_{i}\right)_{N-1}-2}\right) e^{-0.05\left(V_{i}\right)_{N-1}-2.5}}{7\left(1+e^{-0.1\left(V_{i}\right)_{N-1}-2}\right) e^{-0.05\left(V_{i}\right)_{N-1}-2.5}+100}, \\
& \left(d_{i}\right)_{N}=\frac{20+\left(V_{i}\right)_{N-1}}{20+\left(V_{1}\right)_{N-1}+12.5\left(1-e^{-0.1\left(V_{i}\right)_{N-1}-2}\right) e^{-\left(\left(V_{i}\right)_{N-1}+30\right) / 80}}, \\
& \left(\tau_{i}\right)_{N}=\frac{100\left(1+e^{-0.1\left(V_{i}\right)_{N-1}-2}\right)}{r_{n}\left[\left(20+\left(V_{i}\right)_{N-1}\right)+12.5 e^{-\left(\left(V_{i}\right)_{N-1}+30\right) / 80}\left(1+e^{-0.1\left(V_{i}\right)_{N-1}-2}\right)\right]} .
\end{aligned}
$$

To achieve the discrete-time memristive synapse-connected Chay twin-neuron model (7), we employ a low-cost yet powerful XC7Z020 FPGA to execute the model for the first time. The FPGA software program using Verilog language is coded, within which the number of iteration $N=150000$, the sampled interval $i=0.001$, and the coefficients of variation $D_{1}=D_{4}=1$ and $D_{2}=D_{4}=2$ are set up. The parameters $g_{\mathrm{I}}, g_{\mathrm{K}, \mathrm{V}}, k$, and $\varphi_{0}$, which impact the firing activities and synchronous behaviors, are changed by the software program to capture the four typical firing activities, time-domain waveforms, and STSs corresponding to the numerical simulations.

The hierarchical structure of the Verilog HDL program is illustrated in Figure 4. A main controller is set to reset the electro-neuron and to start the iteration form of initial conditions for $V_{i}, n_{i}, C_{i}$, and $\varphi$ while the power is on. The floatingtype operation IPs are contained in the lowest inherent IP layer, which can be instanced to construct our own customized IP. In the customized IP layer, add-, subtract-, multiply-, divide-, and exponent-operators are instanced to construct each function of the right side of continuous-time model (4) (expressed as $f_{V 1}$, $f_{n 1}, f_{C 1} f_{V 2}, f_{n 2}, f_{C 2}$, and $\left.f_{\varphi}\right)$. The intermediate variables $m_{1 \infty}$, $h_{1 \infty}, n_{1 \infty}, m_{2 \infty}, h_{2 \infty}$, and $n_{2 \infty}$ have to be calculated before the calculations of $f_{V}, f_{n}$, and $f_{C}$; thus, a sublayer for intermediate variables is hired in this layer. In the iteration layer, the functions $f_{V 1}, f_{n 1}, f_{C 1} f_{V 2}, f_{n 2}, f_{C 2}$, and $f_{\varphi}$ are transmitted by time multiplex way to compute the intermediate vectors $k_{\left(V_{i}\right) q}, k_{\left(n_{i}\right) q}, k_{\left(C_{i}\right) q}$, and $k_{\varphi q}(i=1,2$ and $q=1,2,3,4)$ (marked as $K_{1}, K_{2}, K_{3}$, and $\left.K_{4}\right)$ in (8), and then the $\left(V_{1}\right)_{N},\left(n_{1}\right)_{N},\left(C_{1}\right)_{N},\left(V_{2}\right)_{N},\left(n_{2}\right)_{N},\left(C_{2}\right)_{N}$, and $\varphi_{N}$ are updated. Finally, an output layer is employed to adjust the output signal for visualizing expediently. The RTL schematic for the digitally electronic twin-neuron network is generated, as shown in Figure 5.

Since the outputs of a FPGA are digital, they are fed to a two-channel 14 bit D/A converter (AD9767) combined with the peripheral circuit to convert the digital outputs into analog ones. Herein, an oscilloscope Agilent DSO-X $3012 \mathrm{~A}$ is employed to display the output analog signals. Note that, all variables are in the single-precision floatingtype during the computation process, so they must be converted into integers and enlarged to the range of $[-8192,8191]$ to take full use of 14 bits of the DAC digital input ports. The time-domain waveforms of $V_{1}$ and $V_{2}$ and 


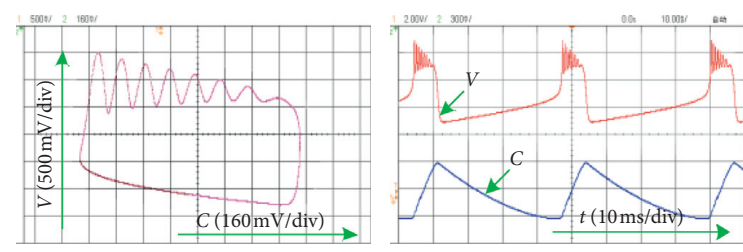

(a)

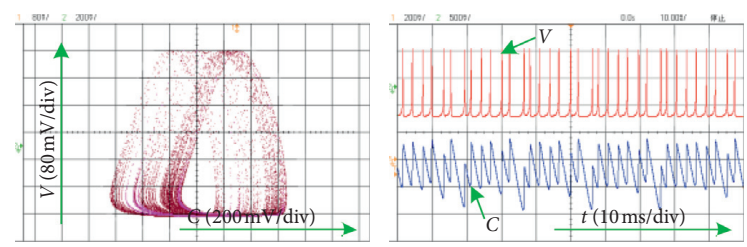

(c)
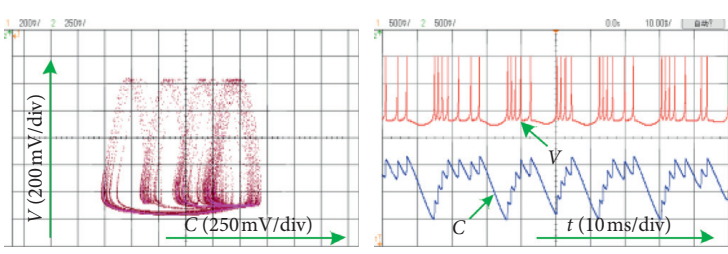

(b)
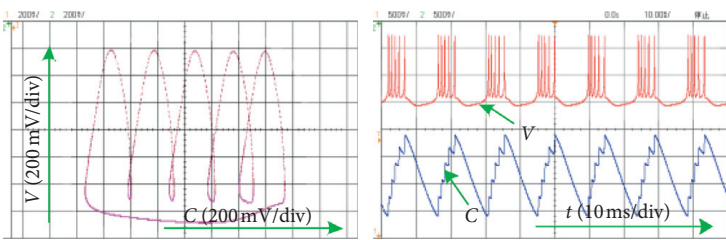

(d)

Figure 7: Experimentally captured trajectories in the $\mathrm{C}-\mathrm{V}$ phase plane and time-domain waveforms. (a) Periodic bursting behavior for $g_{\mathrm{I}}=1250 \mathrm{mS} / \mathrm{cm}^{2}$ and $g_{\mathrm{K}, \mathrm{V}}=1700 \mathrm{mS} / \mathrm{cm}^{2}$; (b) chaotic bursting behavior for $g_{\mathrm{I}}=1850 \mathrm{mS} / \mathrm{cm}^{2}$ and $g_{\mathrm{K}, \mathrm{V}}=1700 \mathrm{mS} / \mathrm{cm}^{2}$; (c) chaotic spiking behavior for $g_{\mathrm{I}}=1925 \mathrm{mS} / \mathrm{cm}^{2}$ and $g_{\mathrm{K}, \mathrm{V}}=1700 \mathrm{mS} / \mathrm{cm}^{2}$; (d) periodic bursting behavior with burster-5 for $g_{\mathrm{I}}=1800 \mathrm{mS} / \mathrm{cm}^{2}$ and $g_{\mathrm{K}, \mathrm{V}}=1650 \mathrm{mS} / \mathrm{cm}^{2}$.

synchronous transition states in the $V_{1}-V_{2}$ plane for different coupling strength $k$ and initial condition $\varphi_{0}$ in the Chay bi-neuron network are captured and displayed in Figure 6. The amplitudes of $V_{1}$ and $V_{2}$ on the oscilloscope are differed from that of MATLAB simulations in Figure 2, but they are proportional with each other due to the same enlargement. It is clearly shown that the proposed FPGAbased digital hardware can verify the synchronous behavior for the memristive synapse-connected Chay twinneuron network. Note that the initial states in our digital circuit experiment are accurately assigned in the software [44]. This is very different from the randomly sensed way of acquiring initial states by repeatedly switching on and off the power supply in an analog circuit experiment [45]. Hence, the initial states are determined in the digital circuits but undetermined in the analog ones.

Besides, a FPGA-based digital hardware electronic neuron is simply realized to confirm the four representative firing activities in the $3 \mathrm{D}$ Chay neuron model. The trajectories in the $V_{1}-V_{2}$ phase plane and time sequences of $V_{1}$ and $V_{2}$ are captured and displayed in Figure 7 . The model parameters are selected the same as these utilized in Figure 1.

\section{Conclusion}

Four kinds of representative firing activities classified on the dependence of two maximal conductances in a 3D Chay neuron model are briefly reviewed. Then, a memristive synapse-connected Chay twin-neuron network is built, upon which synchronous behaviors are explored by utilizing timedomain waveforms, STSs, and MSEs. The numerical simulations demonstrated the success and effectiveness of employing the memristor synapse to achieve synchronization. It is found that, associating with the large coupling strength and more negative initial condition of the memristor, synchronous behaviors are achieved. An effective approach to implement the electronic neuron and the Chay twin-neuron network via FPGA are employed, from which the four kinds of representative firing activities of chaotic and periodic bursting/spiking behaviors, as well as the STSs are experimentally captured to confirm the correctness of the numerical ones. Synchronous behavior disclosed in neuronal network can well reveal the benefit for understanding the dynamical intricacy in the biological neurons and reflect the feasibility of diverse neuron-based applications.

\section{Data Availability}

The data used to support the findings of this study are available from the corresponding author upon request.

\section{Conflicts of Interest}

The authors declare that they have no conflicts of interest.

\section{Acknowledgments}

This work was supported by the National Natural Science Foundation of China under Grant nos. 61801054 and 51777016, Natural Science Foundations of Jiangsu Province, China, under Grant nos. BK20160282 and BK20191451, and Postgraduate Research and Practice Innovation Program of Jiangsu Province, China, under Grant nos. KYCX19_1768 and KYCX20_2550.

\section{References}

[1] F. Parastesh, K. Rajagopal, F. E. Alsaadi, T. Hayat, V.-T. Pham, and I. Hussain, "Birth and death of spiral waves in a network of hindmarsh-rose neurons with exponential magnetic flux and excitable media," Applied Mathematics and Computation, vol. 354, pp. 377-384, 2019.

[2] A. Mauroy and R. Sepulchre, "Clustering behaviors in networks of integrate-and-fire oscillators," Chaos, vol. 18, no. 3, Article ID 037122, 2008.

[3] K. Wu, T. Wang, C. Wang, T. Du, and H. Lu, "Study on electrical synapse coupling synchronization of hindmarsh-rose 
neurons under Gaussian white noise," Neural Computing and Applications, vol. 30, no. 2, pp. 551-561, 2018.

[4] S. Mostaghimi, F. Nazarimehr, S. Jafari, and J. Ma, "Chemical and electrical synapse-modulated dynamical properties of coupled neurons under magnetic flow," Applied Mathematics and Computation, vol. 348, pp. 42-56, 2019.

[5] J. Ma and J. Tang, "A review for dynamics of collective behaviors of network of neurons," Science China Technological Sciences, vol. 58, no. 12, pp. 2038-2045, 2015.

[6] R. D. Pinto, P. Varona, A. R. Volkovskii, A. Szücs, H. D. I. Abarbanel, and M. I. Rabinovich, "Synchronous behavior of two coupled electronic neurons," Physical Review E, vol. 62, no. 2, pp. 2644-2656, 2000.

[7] G. Ren, Y. Xu, and C. Wang, "Synchronization behavior of coupled neuron circuits composed of memristors," Nonlinear Dynamics, vol. 88, no. 2, pp. 893-901, 2017.

[8] Z. T. Njitache, I. S. Doubla, J. Kengne, and A. Cheukem, "Coexistence of firing patterns and its control in two neurons coupled through an asymmetric electrical synapse," Chaos: An Interdisciplinary Journal of Nonlinear Science, vol. 30, no. 2, Article ID 023101, 2020.

[9] A. Tchaptchet, "Activity patterns with silent states in a heterogeneous network of gap-junction coupled huber-braun model neurons," Chaos: An Interdisciplinary Journal of Nonlinear Science, vol. 28, no. 10, Article ID 106327, 2018.

[10] A. Muhammad, K. S. Hong, and M. Y. Jeong, "Synchronization of coupled chaotic fitHugh-nagumo systems," Communications in Nonlinear Science and Numerical Simulation, vol. 17, no. 4, pp. 1615-1627, 2012.

[11] M. Rehan, K.-S. Hong, and M. Aqil, "Synchronization of multiple chaotic FitzHugh-Nagumo neurons with gap junctions under external electrical stimulation," Neurocomputing, vol. 74, no. 17, pp. 3296-3304, 2011.

[12] D. S. Alejandro and G. I. Gonzalo, "Noise-sustained synchronization of electrically coupled FitzHugh-Nagumo networks under counterphase external forcing," Physics Letters A, vol. 380, no. 22-23, pp. 1964-1970, 2016.

[13] H. Dalibor, "Synchronization of two Hindmarsh-Rose neurons with unidirectional coupling," Neural Networks, vol. 40, pp. 73-79, 2013.

[14] J. Juang and Y. H. Liang, "Cluster synchronization in networks of neurons with chemical synapses," Chaos, vol. 24, no. 1, Article ID 013110, 2014.

[15] F. Mormann, T. Kreuz, R. G. Andrzejak, P. David, K. Lehnertz, and C. E. Elger, "Epileptic seizures are preceded by a decrease in synchronization," Epilepsy Research, vol. 53, no. 3, pp. 173-185, 2003.

[16] S. J. G. Lewis and R. A. Barker, "Understanding the dopaminergic deficits in Parkinson's disease: insights into disease heterogeneity," Journal of Clinical Neuroscience, vol. 16, no. 5, pp. 620-625, 2009.

[17] P. J. Uhlhaas and W. Singer, "Neural synchrony in brain disorders: relevance for cognitive dysfunctions and pathophysiology," Neuron, vol. 52, no. 1, pp. 155-168, 2006.

[18] M. Lv, C. Wang, G. Ren, J. Ma, and X. Song, "Model of electrical activity in a neuron under magnetic flow effect," Nonlinear Dynamics, vol. 85, no. 3, pp. 1479-1490, 2016.

[19] M. Lv and J. Ma, "Multiple modes of electrical activities in a new neuron model under electromagnetic radiation," Neurocomputing, vol. 205, pp. 375-381, 2016.

[20] L. Chua, "Everything you wish to know about memristors but are afraid to ask," Radioengineering, vol. 24, no. 2, pp. 319-367, 2015.
[21] L. O. Chua, V. Sbitnev, and H. Kim, "Hodgkin-Huxley axon is made of memristors," International Journal of Bifurcation and Chaos, vol. 22, no. 3, Article ID 1230011, 2012.

[22] B. Bao, Q. Yang, D. Zhu, Y. Zhang, Q. Xu, and M. Chen, "Initial-induced coexisting and synchronous firing activities in memristor synapse-coupled Morris-Lecar bi-neuron network," Nonlinear Dynamics, vol. 99, no. 3, pp. 2339-2354, 2020.

[23] Y. Xu, Y. Jia, J. Ma, A. Alsaedi, and B. Ahmad, "Synchronization between neurons coupled by memristor," Chaos, Solitons \& Fractals, vol. 104, pp. 435-442, 2017.

[24] J. Ma, L. Mi, P. Zhou, Y. Xu, and T. Hayat, "Phase synchronization between two neurons induced by coupling of electromagnetic field," Applied Mathematics and Computation, vol. 307, pp. 321-328, 2017.

[25] F. Xu, J. Zhang, T. Fang, S. Huang, and M. Wang, "Synchronous dynamics in neural system coupled with memristive synapse," Nonlinear Dynamics, vol. 92, no. 3, pp. 1395-1402, 2018.

[26] H. Bao, W. Liu, and A. Hu, "Coexisting multiple firing patterns in two adjacent neurons coupled by memristive electromagnetic induction," Nonlinear Dynamics, vol. 95, no. 1, pp. 43-56, 2019.

[27] T. R. Chay, "Chaos in a three-variable model of an excitable cell," Physica D: Nonlinear Phenomena, vol. 16, no. 2, pp. 233-242, 1985.

[28] A. L. Hodgkin and A. F. Huxley, "A quantitative description of membrane current and its application to conduction and excitation in nerve," Bulletin of Mathematical Biology, vol. 52, no. 1-2, pp. 25-71, 1990.

[29] Z. Yang, Q. Lu, H. Gu, and W. Ren, "Integer multiple spiking in the stochastic Chay model and its dynamical generation mechanism," Physics Letters A, vol. 299, no. 5-6, pp. 499-506, 2002.

[30] H. Gu, M. Yang, L. Li, Z. Liu, and W. Ren, "Dynamics of autonomous stochastic resonance in neural period adding bifurcation scenarios," Physics Letters A, vol. 319, no. 1-2, pp. 89-96, 2003.

[31] Z. Yang, Q. Lu, H. Gu, and W. Ren, "Gwn-induced bursting, spiking, and random subthreshold impulsing oscillation before hopf bifurcations in the Chay model," International Journal of Bifurcation and Chaos, vol. 14, no. 12, pp. 41434159, 2004.

[32] L. Duan, Q. Lu, and Q. Wang, "Two-parameter bifurcation analysis of firing activities in the Chay neuronal model," Neurocomputing, vol. 72, no. 1-3, pp. 341-351, 2008.

[33] Y. Yuan, N. Pang, Y. D. Chen, Y. Wang, and X. L. Li, "Theoretical analysis of the effects of transcranial magnetroacoustical simulation on neuronal firing rhythm and $\mathrm{Ca}^{2+}$ concentration with Chay neuron model," Biomedical Physics \& Engineering Express, vol. 3, Article ID 055006, 2017.

[34] B. C. Bao, A. H. Hu, H. Bao, Q. Xu, M. Chen, and H. G. Wu, "Three-dimensional memristive Hindmarsh-Rose neuron model with hidden coexisting asymmetric behaviors," Complexity, vol. 2018, Article ID 3872573, 11 pages, 2018.

[35] N. Dahasert, İ. Öztürk, and R. Kiliç, "Experimental realizations of the HR neuron model with programmable hardware and synchronization applications," Nonlinear Dynamics, vol. 70, no. 4, pp. 2343-2358, 2012.

[36] H. Soleimani and E. M. Drakakise, "An efficient and reconfigurable synchronous neuron model," IEEE Transactions on Circuits and Systems II: Express Briefs, vol. 65, no. 1, pp. 91-95, 2018. 
[37] M. Nouri, M. Hayati, T. Serrano-Gotarredona, and D. Abbott, "A digital neuromorphic realization of the 2-D Wilson neuron model," IEEE Transactions on Circuits and Systems II: Express Briefs, vol. 66, no. 1, pp. 136-140, 2019.

[38] M. Hayati, M. Nouri, S. Haghiri, and D. Abbott, "Digital multiplierless realization of two coupled biological MorrisLecar neuron model," IEEE Transactions on Circuits and Systems I: Regular Papers, vol. 62, no. 7, pp. 1805-1814, 2015.

[39] M. Nouri, G. R. Karimi, A. Ahmadi, and D. Abbott, "Digital multiplierless implementation of the biological FitzHughNagumo model," Neurocomputing, vol. 165, pp. 468-476, 2015.

[40] V. Ntinas, I. Vourkas, A. Abusleme, G. C. Sirakoulis, and A. Rubio, "Experimental study of artificial neural networks using a digital memristor simulator," IEEE Transactions on Neural Networks and Learning Systems, vol. 29, no. 10, pp. 5098-5110, 2018.

[41] Y.-S. Fan and T. R. Chay, "Generation of periodic and chaotic bursting in an excitable cell model," Biological Cybernetics, vol. 71, no. 5, pp. 417-431, 1994.

[42] Y. Li, H. Gu, and X. Ding, "Bifurcations of enhanced neuronal bursting activities induced by the negative current mediated by inhibitory autapse," Nonlinear Dynamics, vol. 97, no. 4, pp. 2091-2105, 2019.

[43] H. G. Gu and W. W. Xiao, "Different between intermittent chaotic bursting and spiking of nural firing patterns," International Journal of Bifurcation and Chaos, vol. 24, no. 6, Article ID 1450082, 2014.

[44] F. Yu, L. Liu, B. He et al., "Analysis and FPGA realization of a novel 5D hyperchaotic four-wing memristive system, active control synchronization, and secure communication application," Complexity, vol. 2019, Article ID 4047957, 18 pages, 2019.

[45] Q. Xu, Q. L. Zhang, N. Wang, H. G. Wu, and B. C. Bao, "An improved memristive diode bridge-based band pass filter chaotic circuit," Mathematical Problems in Engineering, vol. 2017, Article ID 2461964, 11 pages, 2017. 